\title{
EEG activity as an objective measure of cognitive load during effortful listening: A study on pediatric subjects with bilateral...
}

Article · May 2017

DOI: 10.1016/j.ijporl.2017.05.006

CITATIONS

0

8 authors, including:

\section{Giulia Cartocci}

Sapienza University of Rome 22 PUBLICATIONS 105 CITATIONS SEE PROFILE

\section{Anton Giulio Maglione}

Sapienza University of Rome 46 PUBLICATIONS 230 CITATIONS SEE PROFILE
READS

39

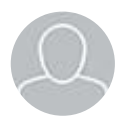

Sara Giannantonio

Catholic University of the Sacred Heart 26 PUBLICATIONS 138 CITATIONS

SEE PROFILE

\section{Ambra Brizi}

Sapienza University of Rome

13 PUBLICATIONS 7 CITATIONS

SEE PROFILE

Some of the authors of this publication are also working on these related projects: 


\title{
EEG activity as an objective measure of cognitive load during effortful listening: A study on pediatric subjects with bilateral, asymmetric sensorineural hearing loss
}

\author{
Pasquale Marsella a , Alessandro Scorpecci a, *, Giulia Cartocci ${ }^{\mathrm{b}}$, Sara Giannantonio ${ }^{\text {a }}$, \\ Anton Giulio Maglione ${ }^{\mathrm{b}}$, Isotta Venuti ${ }^{\mathrm{c}}$, Ambra Brizi ${ }^{\mathrm{d}}$, Fabio Babiloni ${ }^{\mathrm{b}}$ \\ ${ }^{a}$ Audiology and Otosurgery Unit, Bambino Gesù Pediatric Hospital, Italy \\ ${ }^{\mathrm{b}}$ Department of Molecular Medicine, "Sapienza" University of Rome, Italy \\ ${ }^{\mathrm{c}}$ BrainSigns srl, Rome, Italy \\ d Department of Developmental and Social Psychology, "Sapienza" University of Rome, Italy
}

\section{A R T I C L E I N F O}

\section{Article history:}

Received 11 February 2017

Received in revised form

12 May 2017

Accepted 13 May 2017

Available online 17 May 2017

\section{Keywords:}

Listening effort

Cognitive load

Children

Hearing loss

Electroencephalography

Alpha

\begin{abstract}
A B S T R A C T
Objectives: Deaf subjects with hearing aids or cochlear implants generally find it challenging to understand speech in noisy environments where a great deal of listening effort and cognitive load are invested. In prelingually deaf children, such difficulties may have detrimental consequences on the learning process and, later in life, on academic performance. Despite the importance of such a topic, currently, there is no validated test for the assessment of cognitive load during audiological tasks. Recently, alpha and theta EEG rhythm variations in the parietal and frontal areas, respectively, have been used as indicators of cognitive load in adult subjects.

The aim of the present study was to investigate, by means of EEG, the cognitive load of pediatric subjects affected by asymmetric sensorineural hearing loss as they were engaged in a speech-in-noise identification task.

Methods: Seven children ( $4 \mathrm{~F}$ and $3 \mathrm{M}$, age range $=8-16$ years) affected by asymmetric sensorineural hearing loss (i.e. profound degree on one side, mild-to-severe degree on the other side) and using a hearing aid only in their better ear, were included in the study. All of them underwent EEG recording during a speech-in-noise identification task: the experimental conditions were quiet, binaural noise, noise to the better hearing ear and noise to the poorer hearing ear. The subjects' Speech Recognition Thresholds (SRT) were also measured in each test condition. The primary outcome measures were: frontal EEG Power Spectral Density (PSD) in the theta band and parietal EEG PSD in the alpha band, as assessed before stimulus (word) onset.

Results: No statistically significant differences were noted among frontal theta power levels in the four test conditions. However, parietal alpha power levels were significantly higher in the "binaural noise" and in the "noise to worse hearing ear" conditions than in the "quiet" and "noise to better hearing ear" conditions $(\mathrm{p}<0.001)$. SRT scores were consistent with task difficulty, but did not correlate with alpha and theta power level variations.

Conclusion: This is the first time that EEG has been applied to children with sensorineural hearing loss with the purpose of studying the cognitive load during effortful listening. Significantly higher parietal alpha power levels in two of three noisy conditions, compared to the quiet condition, are consistent with increased cognitive load. Specifically, considering the time window of the analysis (pre-stimulus), parietal alpha power levels may be a measure of cognitive functions such as sustained attention and selective inhibition. In this respect, the significantly lower parietal alpha power levels in the most challenging listening condition (i.e. noise to the better ear) may be attributed to loss of attention and to the subsequent fatigue and "withdrawal" from the task at hand.
\end{abstract}

(c) 2017 Elsevier B.V. All rights reserved.

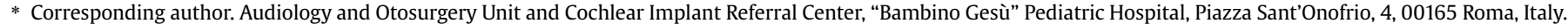
E-mail address: alessandroscorpecci@yahoo.it (A. Scorpecci).
} 


\section{Introduction}

People who are hard of hearing and use hearing aids (HA) or cochlear implants $(\mathrm{CI})$ often complain about being tired from straining to hear, especially in the presence of noisy environments or multiple talkers. Self-reports of listening effort and fatigue are very common even among "star" patients who score particularly well in speech recognition tests, both in quiet and in noise, yet at the cost of deploying a great deal of cognitive resources [1]. A frequent consequence of such difficulties is early exhaustion, discouragement and ultimately withdrawal from social life, where the ability to listen in challenging conditions is often required.

In children and adolescents with sensorineural hearing loss, the effect of prolonged effortful listening is likely to be even more detrimental, impairing the acquisition of linguistic abilities and being an obstacle to achieving satisfactory academic performance [2].

Given these premises, it is clear that reliable measures of the listening effort experienced by hearing-impaired subjects would enable to investigate an issue of crucial importance, whose assessment is currently beyond the possibility of conventional audiometric tests. If available for clinical use, such measures could have important implications [3]: first, they would help clinicians in decision-making concerning the most appropriate treatment of hearing loss (e.g. HA vs. CI); secondly, they would allow a more comprehensive assessment of functional outcomes, especially in cases where treatment is aimed at restoring binaural hearing, such as asymmetrical hearing loss or single-sided deafness [4].

Despite the growing importance of this topic, the assessment of effortful listening still represents a challenge for a number of reasons. In the first instance, there is currently no shared general definition of this concept; secondly, and subsequently, there is no agreement as to which outcome measures should be applied; thirdly, very little is known about age-related variations of the proposed outcome measures and, more specifically, about how they should be applied to pediatric subjects.

In a recent consensus paper, listening effort was generally defined as "deliberate allocation of mental resources to overcome obstacles in goal pursuit when carrying out a listening task" [5], and in the frame of an established limited-capacity resource model [6]. In this model, a subject's cognitive resources are limited; while listening, a subject deliberately allocates a part of such resources to the task, depending on the inherent demands: when demands increase, due to environmental factors (acoustically unfavorable environment), subject-related factors (e.g. hearing impairment or poor knowledge of a language) or talker-related factors (poorly intelligible accent or timbre), the amount of cognitive load invested in the task will also increase. Therefore in this paper, we will include the listening effort in the more general concept of cognitive load.

So far, attempts at assessing cognitive load in audiological settings have been conducted on adult subjects, by means of selfreports, behavioral methods and physiological measures. Physiological measures seem very promising in that they are objective, where they are used to quantify effort in terms of a set of physiological responses to demanding conditions and allow a real-time assessment. Besides autonomic nervous system activity indexes such as pupil dilation [7] and heart rate variability [8], electroencephalographic (EEG) activity has also been studied in relation with mental effort, in which case alpha $(8-12 \mathrm{~Hz})$ power in the parietal cortex and theta $(1-4 \mathrm{~Hz})$ power in the frontal cortex have been found to be influenced by cognitive performances [9] and, more specifically, to increase during effortful listening tasks [10-12]. EEG alpha and theta rhythms have been investigated as a tool to estimate the cognitive load in hearing impaired participants in preliminary studies on adult subjects, while using different $\mathrm{CI}$ processors and features $[13,14]$.

In designing the present study, we attempted to answer the following question:

Is it possible to apply Electroencephalography (EEG) to obtain an objective measure of cognitive load during effortful listening in pediatric subjects with sensorineural hearing loss?

For the assessment of the impact of hearing loss upon cognitive load, we chose fully cooperative children and adolescents who had asymmetric sensorineural hearing loss (i.e. profound degree in the poorer ear and ranging from a mild to a severe degree on the better contralateral ear) and were unilateral HA users only in their better hearing ear, in a within-subject design.

Aim of this investigation is, thus, to explore, in a pediatric sample, how pre-stimulus alpha and theta EEG activity levels are modified in the different listening conditions.

Specifically, we hypothesized that parietal alpha and frontal theta EEG activity should vary consistently with the degree of cognitive load required by listening, leading to both parietal alpha and frontal theta power being higher in the most difficult listening conditions.

\section{Material and methods}

The procedures described in the present study were reviewed and approved by the institutional Ethic Committee (Protocol: 705/ FS) and were in accordance with the Helsinki declaration. Parents of the subjects provided a written informed consent for their child's participation to the investigation.

\subsection{Population}

In order to identify subjects that could suit our paradigm, the following inclusion criteria were set: age $\geq 8$ years and $<18$ years, both genders, normal reading abilities and normal verbal IQ, presence of an asymmetric hearing loss, defined as profound degree on one side (average threshold for pure tone frequencies $250-4000 \mathrm{~Hz} \geq 90 \mathrm{~dB} \mathrm{HL}$ ) and a mild to severe degree on the other side (i.e. average threshold for pure tone frequencies $250-4000 \mathrm{~Hz}$ better than contralateral ear by at least $10 \mathrm{~dB} \mathrm{HL}$ ), disyllabic word recognition score in quiet $\geq 70 \%$. The lower age limit of 8 years was set to ensure that participants had full achievement of reading abilities, and also because EEG activity is not considered mature in younger children $[9,15]$. At the moment of the study, all of the subjects had been using for at least one year a digital HA at its best fitting only in their better hearing ear, because they did not perceive any measurable benefit from amplification in the poorer ear. This particular configuration for hearing loss and use of amplification permitted a within-subject study design in which each patient acted as their own control for the comparative measures employed.

Seven subjects meeting the requirements $(4 \mathrm{~F}, 3 \mathrm{M}$, age range 8-16 years) were included in the study. Their main demographic and clinical characteristics, together with their audiometric pure tone average (PTA) and word recognition score in quiet, are detailed in Table 1.

\subsection{Outcome measures and stimuli conditions}

The primary outcome measures were theta and alpha power levels (Power Spectral Density, PSD) of EEG, estimated for the frontal and parietal cortex, respectively, based on existing literature [9-12].

Subjects performed a forced-choice word identification task, in which stimuli consisted of Italian disyllabic words from 
Table 1

Demographic and clinical characteristics of the study patients.

\begin{tabular}{|c|c|c|c|c|c|c|}
\hline Patient & Gender & $\begin{array}{l}\text { Age } \\
\text { (years) }\end{array}$ & Etiology of hearing loss & $\begin{array}{l}0.25-4 \mathrm{kHz} \text { PTA in } \\
\text { better ear }\end{array}$ & $\begin{array}{l}0.25-4 \mathrm{kHz} \text { PTA in } \\
\text { poorer ear }\end{array}$ & $\begin{array}{l}\text { Word recognition score (\%) in } \\
\text { quiet }\end{array}$ \\
\hline C.D. & M & 14 & $\begin{array}{l}\text { Late-onset, progressive hearing loss following congenital } \\
\text { CMV infection }\end{array}$ & 85 (left) & 110 (right) & 100 \\
\hline H.M.E. & $\mathrm{F}$ & 11 & Prematurity: born at 24 weeks, 4 months of $\mathrm{NICU}^{\mathrm{a}}$ & 78 (right) & 105 (left) & 70 \\
\hline G.L. & M & 16 & Prematurity: born at 28 weeks, 2 months of NICU ${ }^{a}$ & 81 (left) & 118 (right) & 100 \\
\hline S.V.A. & $\mathrm{F}$ & 11 & Progressive hearing loss of unknown etiology & 73 (right) & 111 (left) & 80 \\
\hline C.I. & $\mathrm{F}$ & 10 & VACTERL $^{\mathrm{b}}$ association & 82 (right) & 104 (left) & 80 \\
\hline S.G. & $\mathrm{F}$ & 13 & unknown & 60 (right) & 110 (left) & 90 \\
\hline C.L. & M & 8 & unknown & 75 (right) & 103 (left) & 100 \\
\hline
\end{tabular}

Neonatal Intensive Care Unit.

b Acronym standing for: Vertebral defects, Anal atresia, Cardiac defects, Tracheo-esophageal fistula, Renal anomalies and Limb abnormalities.

"Audiometria Vocale GNResound" [16], delivered free-field at an intensity of $65 \mathrm{~dB}$ HL by a loudspeaker set $1 \mathrm{~m}$ in front of the patient. Continuous 4-talker babble background noise was used as the competing signal in order to provide informational masking, i.e. masking containing semantic information highly interfering with the linguistic processing of the target. Test conditions were Quiet and three background noise configurations that have already been used in reported studies investigating the benefits of binaural hearing [17-19], where the competing noise was delivered: (1) binaurally, i.e. by two loudspeakers angled at $-45^{\circ}$ and $+45^{\circ}$; (2) at an angle of $+90^{\circ}$ from the signal; (3) at an angle of $-90^{\circ}$ from the signal. During EEG recordings, the noise was kept constant at $55 \mathrm{~dB}$ $\mathrm{HL}(\mathrm{SNR}=+10)$.

Given the peculiar audiological configuration of the study cohort (asymmetric hearing loss), the experimental conditions were labeled as: $\mathbf{Q}$ (Quiet); $\mathbf{N}_{\mathbf{B}}$, when the noise was delivered binaurally, thus reaching both ears with equal loudness and timing; N NorSE EAR, when the noise was delivered to the worse ear side; $\mathbf{N}_{\mathbf{B E T T E R}}$ EAR, when the noise was delivered to the better ear side.

For EEG recordings, a digital outpatient monitoring system (Bemicro EBNeuro, Italy) was used. Subjects were seated on a comfortable chair in a soundproof room. For the measurements, a 19-channel electrode cap was used. Signals were measured with a 256-Hz sampling frequency and collected simultaneously during the experiment. A $50-\mathrm{Hz}$ notch filter was applied to remove electrical interference from the power line. A ground and a reference electrode were placed on the subject's forehead and electrode impedances were maintained below $10(\mathrm{k} \Omega)$. The EEG recording was filtered with a band-pass filter $(2-30 \mathrm{~Hz})$. The Independent Component Analysis and an artifact removal algorithm were applied to reject trials presenting eye-blink components or head movements, respectively.

For each word stimulus, the subjects were asked to listen and to read the target word presented among the four possible options provided; one second after the stimulus offset, the words appeared on an initially blank laptop screen set in front of the subjects. Each response option was placed in a different colored box on the screen. For response, the subject was instructed to select the correct word by pressing the corresponding color button on a color-coded keyboard customized for the investigation. Each target word had an equal probability (25\%) of being in one of the four possible colored boxes and positions.

Each experimental listening condition included 20 trials of 20 word stimuli, each lasting for maximum $8 \mathrm{~s}$, varying in length depending on the child's response time. In each trial, the onesecond time segment immediately preceding the word stimulus onset was selected for EEG analysis.

For each segmental interval the Power Spectrum Density (PSD) was calculated, observing the EEG PSD values in theta band $(4-8 \mathrm{~Hz})$ over the central frontal area $(\mathrm{F} 7, \mathrm{~F} 8, \mathrm{~F} 3, \mathrm{~F} 4, \mathrm{Fz})$ and in alpha band $(8-12 \mathrm{~Hz})$ over the central parietal area $(\mathrm{P} 7, \mathrm{P} 8, \mathrm{P} 3, \mathrm{P} 4, \mathrm{Pz})$. For estimation of the cortical activity all the 19 recording channels were used, according to the 10-20 international system, in order to solve the linear inverse problem.

Cortical activity from EEG scalp recordings was reconstructed using a realistic head model (Colin Template provided by the McGill University) corresponding to 6239 voxels at a $5 \times 5 \times 5 \mathrm{~mm}$ spatial resolution uniformly distributed on the cortical gray matter. The difference in source localization of cortical oscillations between groups in each frequency band was assessed by means of voxel-byvoxel independent sample F-ratio-tests, based on eLORETA logtransformed current density power. In the resulting statistical three-dimensional images, a statistical nonparametric permutation test was used to identify cortical voxels showing significant differences. This test, described by Nichols and Holmes [20], employs a randomization strategy to determine the critical probability threshold values for the actually observed $t$ values, with correction for multiple comparisons across all voxels and all frequencies. In the present study, a total of 5000 permutations were used to determine significance for each randomization test. The use of statistical nonparametric maps applied to LORETA images has been validated in several studies [21-23].

For each subject, a Speech Recognition Threshold (SRT) score was also obtained, defined as the Signal-to-Noise Ratio (SNR) level at which a subject responds $50 \%$ correct. For its assessment, a disyllabic word recognition task in babble noise was used, with the above described material [16], adopting the established psychoacoustic up-and-down procedure [24,25], in which the signal is kept constant at $65 \mathrm{~dB}$ HL and the SNR is lowered by $2 \mathrm{~dB}$ HL if the subject repeats the word correctly, or is increased by $2 \mathrm{~dB}$ HL for each incorrect response. The one-down one-up procedure allows an estimate of the $50 \%$ correct point on the psychometric function, SRT being calculated as the arithmetic mean of the SNRs of the last 10 reversals out of a total of 12 reversals. The noise configurations $\left(\mathrm{N}_{\mathrm{B}}, \mathrm{N}_{\text {WORSE EAR, }} \mathrm{N}_{\mathrm{BETTER}}\right.$ EAR $)$ were exactly the same as used for the EEG recordings stated above. So as to minimize order effects, noise conditions were presented randomly in both EEG and SRT measurements.

\subsection{Statistical analysis}

Repeated measures one-way Analysis of Variance (ANOVA) statistics was used to compare results across the different noise conditions for the tasks (Statistica 8, Dell). Post hoc comparisons were performed using the Duncan test, while simple and partial correlation analysis was conducted by means of Pearson's " $r$ " correlation coefficient. P values $<0.05$ were accepted as statistically significant.

\section{Results}

As expected, SRT scores with babble noise were higher in the 
most difficult noise configuration, i. e. $\mathrm{N}_{\text {BETTER EAR }}(16.4 \pm 5.4 \mathrm{~dB} \mathrm{HL})$, and lower in the relatively easier conditions, i.e. $\mathrm{N}_{\mathrm{B}}(9.9 \pm 7.3 \mathrm{~dB} \mathrm{HL})$ and N NORSE EAR $(8 \pm 5.7 \mathrm{~dB} \mathrm{HL})$. Individual SRT scores in the different conditions are detailed in Table 2.

As described in the Methods, both alpha and theta Power Spectral Density levels were analyzed in the pre-stimulus phase of EEG recordings.

Analysis of results in the theta frequency band of the EEG (mean power for each word in the four conditions $=20 * 4=80$ observations), yielded no significant effect of "condition" on power ( $\mathrm{F}(3$, $57)=2.33, p<0.083$ ), with a tendency to greater power levels in the three noise configurations and to lower power levels in the "Quiet" condition, as showed in Fig. 1. Individual data for theta activity are provided in Table 3.

When EEG activity in the alpha band was considered, there was a significant effect of "condition" on power values $(F(3,57)=34.22$, $\mathrm{p}<0.0001$ ). In this case (Fig. 2), post-hoc Duncan test detected a significant difference between the conditions: $\mathrm{N}_{\mathrm{B}} \mathrm{VS} \mathrm{Q}: \mathrm{p}=0.0001$; $\mathrm{N}_{\mathrm{B}}$ vS $\mathrm{N}_{\text {BETTER EAR }}$ : $\mathrm{p}<0.0001$; Q vs $\mathrm{N}_{\text {WORSE EAR }} \mathrm{p}<0.0001$; $\mathrm{N}_{\text {WORSE }}$ EAR VS N NETTER EAR: $p<0.0001$. Overall, uppermost alpha power levels were observed in the conditions $\mathrm{N}_{B}$ and $\mathrm{N}_{\text {WORSE EAR, whereas }}$

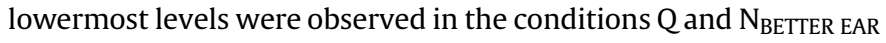
(see Table 4 for individual data).

A correlation analysis run between EEG PSD values and SRT scores in the alpha and theta frequency bands did not yield statistical significance (Pearson's $r=0.02, p=0.6$, and Pearson's $\mathrm{r}=0.16, \mathrm{p}=0.5$, respectively). However, there was a significant positive correlation between alpha and theta PSD levels (Pearson's $\mathrm{r}=0.77, \mathrm{p}<0.001$ ), which remained unchanged after running a partial correlation analysis in which SRT was considered as the control covariate.

Statistical cortical maps were reconstructed only for alpha activity, because this was the only outcome variable which was found to differ significantly across experimental conditions. Fig. 3 illustrates the diffuseness and the lateralization of alpha response in each noise configuration, compared to the baseline quiet condition.

\section{Discussion}

This is the first time that EEG has been applied in children to investigate frontal theta and parietal alpha power levels of EEG in relation to cognitive load during an audiological task. In particular, the study focused on the pre-stimulus phase, i.e. the time interval when noise is on and the subject is waiting for the word to be delivered. The main findings of this research indicate that: 1) frontal theta power did not reveal a significant increase in adverse listening conditions compared to the listening-in-quiet condition; 2) parietal alpha power increased significantly in only some of the more adverse listening conditions compared to the listening-inquiet condition.

Going back to the study hypothesis, we found that only alpha

Table 2

Individual SRT levels for each stimulus configuration.

\begin{tabular}{llll}
\hline Patient & $\mathrm{SRT}_{\mathrm{B}}{ }^{*}$ & $\begin{array}{l}\mathrm{SRT}^{*} \\
\mathrm{~N}_{\text {BETTER EAR }}\end{array}$ & $\begin{array}{l}\mathrm{SRT}^{*} \\
\mathrm{~N}_{\text {WORSE EAR }}\end{array}$ \\
\hline C.D. & 8.8 & 17.2 & 4,8 \\
H.M.E. & 23 & 25 & 16 \\
G.L. & 4 & 16.8 & 3.6 \\
S.V.A. & 9.8 & 12 & 3.8 \\
C.I. & 0 & 20 & 13.2 \\
S.G. & 13.8 & 8 & 2 \\
C.L. & 9.7 & 16 & 12.6 \\
\hline
\end{tabular}

* Speech Recognition Threshold in dB HL. power met expectations that it would be higher in the most difficult listening conditions, whereas theta power showed no significant variations.

An increase in theta power is unanimously considered as an electrophysiological marker of engagement in a task [26]. Over the years, it has been studied in relationship to cognitive load, excitation and interest, high attentional demands, task difficulty, cognitive and mental efforts [27-29]. The finding of non-significant changes for theta power throughout the various listening configurations assessed in this research may seem contradictory to that reported in the existing literature. A possible interpretation would be to assume that the task was not exciting enough for the subjects, and a higher degree of mental effort would have been necessary to elicit significant variations in theta band. Another explanation concerning why theta power levels did not increase could be that in the present study the pre-stimulus interval (i.e. the interval before word onset) was analyzed. In this "pre-word" phase, subjects were only hearing the competing babble noise, anticipating of the test signal but not actively engaged in decoding a test sound signal. In a recent experiment on normal-hearing subjects conducted with an auditory oddball task, Wisniewski et al. [30] found no significant theta variations either before word onset or during "passive" exposure to stimuli, namely as subjects were engaged in another activity. Thus, the pre-stimulus phase of our study may be equivalent to the passive exposure described in Wisniewski's work, where subjects were asked not to pay attention to background noise, but to focus on the incoming word stimuli.

However, the significant within-subject correlation found between frontal theta and parietal alpha power levels suggests that theta activity could be a potential indicator of cognitive resource deployment in effortful audiological tasks, providing a higher degree of effort is required or a different epoch (e.g. during or poststimulus) is analyzed.

The main finding of the present study was the significant increase of parietal alpha power in two of the three most challenging listening conditions, i.e. binaural noise and noise delivered to the worse hearing ear. This verified the study hypothesis concerning alpha power only partially, since the expected rise in the acoustically most adverse condition, where noise was delivered to the better ear, was not observed.

Such results need to be interpreted in the light of the large amount of literature investigating the general relevance of alpha activity and its possible generators, bearing in mind that only a few studies have focused specifically on the impact of various listening tasks.

The earliest research investigating the behavior of parietal alpha power in the pre-stimulus phase was conducted using visual stimuli. Parieto-occipital alpha power was shown to increase as tested subjects are required to direct attention to a relevant stimulus while neglecting other task-irrelevant stimuli, such as portions of space containing distractor information [31], colors, or motions [32]. Other studies have even demonstrated that selective increases of alpha activity occur retinotopically over areas of the parietooccipital cortex where distracting visual stimuli are likely to be processed $[33,34]$. Also works using a double modality of stimulus presentation (e.g. visual vs auditory) have confirmed that alpha power increases as tested subjects try to suppress one modality in favor of another one [35-37].

When purely auditory tasks were used, alpha power in the parietal and occipital cortex was once again found to increase as subjects were preparing for expected auditory stimuli [38,39]. A lateralization of alpha band activity was also observed, whereby alpha power increases over the parietal cortex contralateral to the to-be-ignored stimulus [38].

Our results with the "binaural noise" $\left(\mathrm{N}_{\mathrm{B}}\right)$ and "noise to the 


\section{EEG Theta activity}

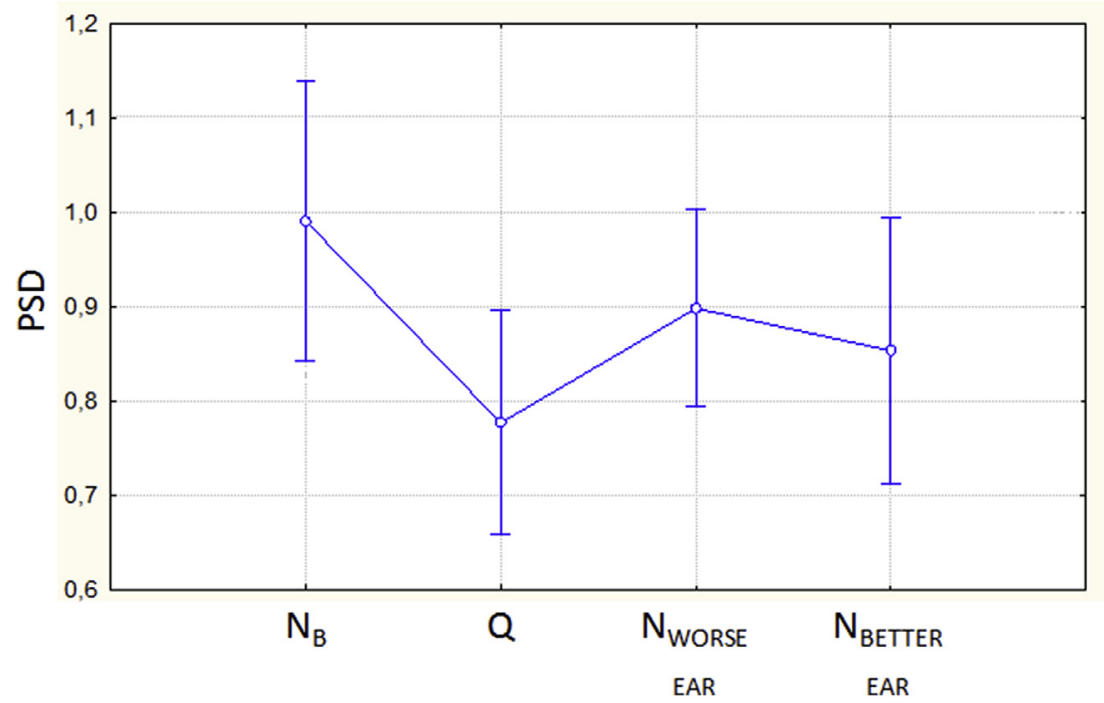

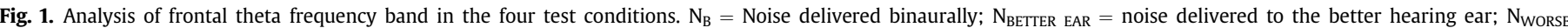

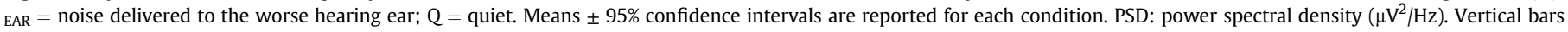
denote 0.95 confidence intervals.

Table 3

Mean PSD levels of frontal theta activity in each stimulus condition.

\begin{tabular}{lllll}
\hline Patient & $\mathrm{Q}^{*}$ & $\mathrm{~N}_{\mathrm{B}}^{*}$ & $\mathrm{~N}_{\text {BETTER EAR }}^{*}$ & $\mathrm{~N}_{\text {WORSE EAR }}^{*}$ \\
\hline C.D. & 2,15 & 2,36 & 2,89 & 1,52 \\
H.M.E. & 0,43 & 1,01 & 0,46 & 0,98 \\
G.L. & 0,17 & 0,24 & 0,15 & 0,16 \\
S.V.A. & 0,29 & 0,35 & 0,31 & 0,34 \\
C.I. & 0,23 & 0,34 & 0,24 & 0,33 \\
S.G. & 1,27 & 0,93 & 0,84 & 1,89 \\
C.L. & 0,90 & 1,69 & 1,13 & 1,06 \\
\hline
\end{tabular}

* PSD values are given as $\mu \mathrm{V}^{2} / \mathrm{Hz}$.
Table 4

Mean PSD levels of parietal alpha activity in each stimulus condition.

\begin{tabular}{lllll}
\hline Patient & $\mathrm{Q}^{*}$ & $\mathrm{~N}_{\mathrm{B}}^{*}$ & $\mathrm{~N}_{\text {BETTER EAR }}^{*}$ & $\mathrm{~N}_{\text {WORSE EAR }}^{*}$ \\
\hline C.D. & 0,82 & 4,76 & 0,62 & 5,66 \\
H.M.E. & 0,41 & 0,64 & 0,57 & 0,38 \\
G.L. & 0,31 & 0,61 & 0,29 & 0,29 \\
S.V.A. & 0,44 & 0,53 & 0,55 & 0,45 \\
C.I. & 0,53 & 0,55 & 0,52 & 0,52 \\
S.G. & 1,28 & 0,80 & 1,39 & 0,89 \\
C.L. & 0,93 & 0,86 & 0,70 & 0,74 \\
\hline
\end{tabular}

* PSD values are given as $\mu \mathrm{V}^{2} / \mathrm{Hz}$.

\section{EEG Alpha activity}

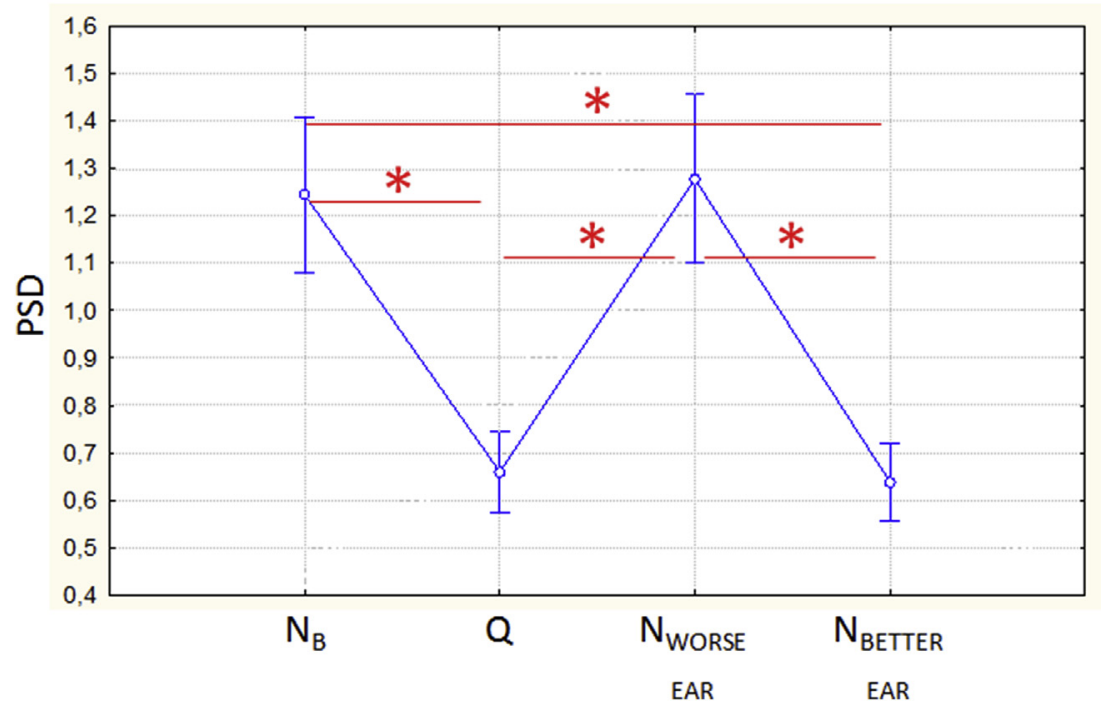

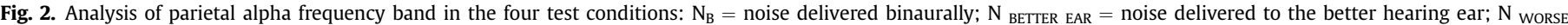

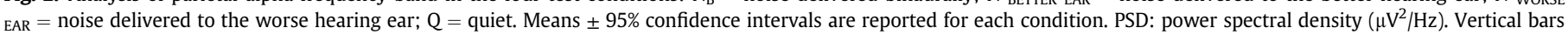
denote 0.95 confidence intervals. 


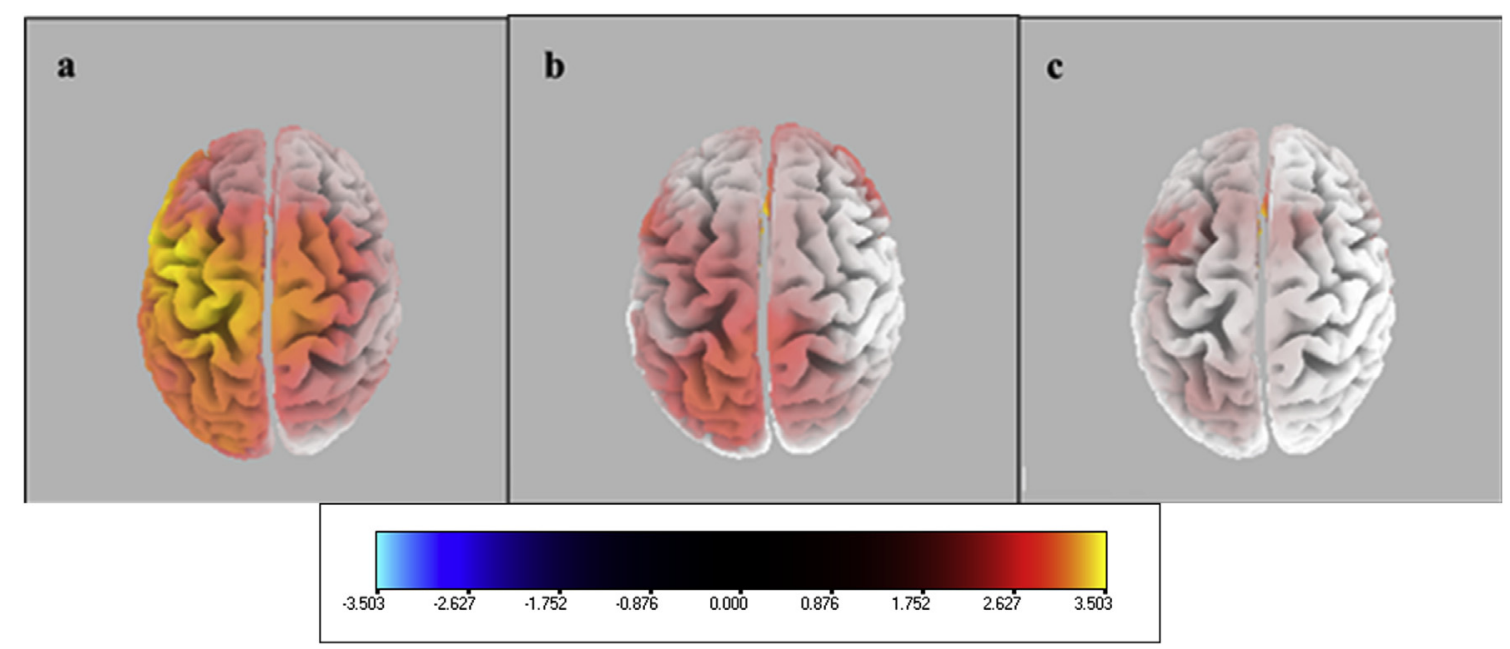

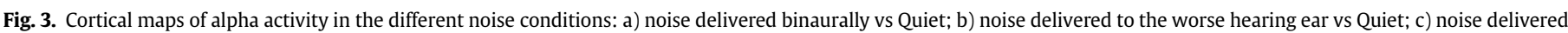

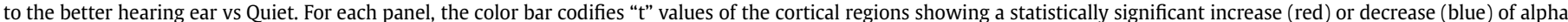

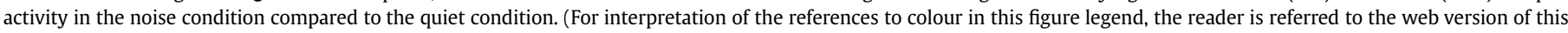
article.)

worse ear" (N NORSE EAR $)$ listening conditions seem to fit the model, suggesting that increases in the parietal alpha power can be an indicator of attentional suppression even in a pediatric sample. In the present study, words were the relevant auditory stimulus and babble noise was irrelevant to the task and thus a to-be-ignored stimulus or distractor.

Overall, this is consistent with recent theories attributing taskrelated increases of alpha activity to the activation of a supramodal functional inhibition system [40-44], i.e. a "top-down" gating system where inhibitory neural pathways eliminate task-irrelevant stimuli and constrain potentially erroneous behaviors.

To the best of our knowledge, this is the first time that a drop in alpha power has been observed in the most challenging listening condition tested (noise was delivered to the better hearing ear). As stated above, under this circumstance our study hypothesis was not fulfilled, whereby alpha power fell to the level of that observed in the quiet test condition. Possibly, this is the reason why no correlation could be found between alpha power levels and SRT scores: whereas average SRT scores decreased with increasing difficulty of the task (i.e. from Quiet to $\mathrm{N}_{\mathrm{BETTER}} \mathrm{EAR}$ ), alpha power levels increased from Quiet to NWORSE EAR, but then decreased again in the $\mathrm{N}_{\text {BETTER EAR }}$ condition.

This "alpha drop phenomenon" may be explained by subjects mentally withdrawing from the task, when it became exceedingly difficult [11]. In their "Framework for Understanding Effortful Listening" model, Pichora Fuller et al. [5] postulate that listening effort increases as task demands increase as long as the subject is motivated toward the task itself, up to a point where task demands exceed the subject's cognitive resources. When this occurs, the subject loses motivation and stops deploying these resources in the task. Specifically, it is likely that the "noise to better ear condition" was too difficult for the subjects selected in the study, whose SRT in that condition was on average $15 \mathrm{~dB}$, that is to say well above the +10 SNR level at which the EEG recordings took place. In this case, it is plausible that the patients stopped directing their attention selectively toward the presented words and completely lost their overall "engagement" in the task. A support to this interpretation comes from the work by Wizniewski [39], in which alpha power levels were compared in normal-hearing adults during an active listening and a passive exposure experiment: during the passive experiment, alpha power levels dropped to rest values.
Thus, it is plausible that in our subjects loss of interest in a too difficult task may have equaled the "passive exposure" condition in Wizniewski's study [39].

Overall, our results suggest that EEG alpha activity derived from the parietal cortex can be used in pediatric subjects with sensorineural hearing loss as an objective measure of cognitive load during effortful listening. In particular, since the pre-stimulus phase was analyzed, sustained attention and selective inhibition seem to be the likeliest behavioral correlates of the observed alpha variations. If confirmed on larger samples, the alpha power drop characterizing the most adverse listening condition suggests that cognitive withdrawal from a task can also be measured objectively.

Even if consistent with recent works in the literature, the results of our study should be interpreted with caution and be supported by further investigations in larger cohort datasets.

Firstly, our data refer to a pediatric population in whom the brain is notoriously immature and, as a consequence, normative data is age dependent. Most of the literature, on the contrary, reports on adult or elderly subjects. For example, it is known that alpha frequency itself increases in a non-linear way [45], whereas global alpha and theta power increase and decrease, respectively, from childhood to puberty [15].

Secondly, the lack of normative data from normal-hearing subjects makes it impossible, at this point, to use absolute alpha and theta power levels to quantify cognitive load.

However, if confirmed by further research on diverse and larger samples, these findings could be used to refine indications for hearing aid or cochlear implant treatment of sensorineural hearing loss, and for a more in-depth assessment of the audiological outcomes of listening devices.

\section{Conflicts of interest}

None.

\section{References}

[1] A.A. Zekveld, S.E. Kramer, J.M. Festen, Cognitive load during speech perception in noise: the influence of age, hearing loss, and cognition on the pupil response, Ear Hear 32 (2011) 498-510.

[2] C. Yoshinaga-Itano, A.L. Sedey, D.K. Coulter, A.L. Mehl, Language of early-and later-identified children with hearing loss, Pediatrics 102 (1998) 1161-1171. 
[3] R. McGarrigle, K.J. Munro, P. Dawes, A.J. Stewart, D.R. Moore, I.G. Barry, S. Amitay, Listening effort and fatigue: what exactly are we measuring? A British society of audiology cognition in hearing special interest group 'white paper', Int. J. Audiol. 53 (2014) 433-440.

[4] J. van Schoonhoven, M. Schulte, M. Boymans, K.C. Wagener, W.A. Dreschler B. Kollmeier, Selecting appropriate tests to assess the benefits of bilateral amplification with hearing aids, Trends Hear 20 (2016) 1-16.

[5] M.K. Pichora-Fuller, S.E. Kramer, M.A. Eckert, B. Edwards, B.W. Hornsby, L.E. Humes, U. Lemke, T. Lunner, M. Matthen, C.L. Mackersie, G. Naylor, N.A. Phillips, M. Richter, M. Rudner, M.S. Sommers, K.L. Tremblay, A. Wingfield, Hearing impairment and cognitive energy: the framework for understanding effortful listening (FUEL), Ear Hear 37 (2016) 5S-27S.

[6] D. Kahneman, Attention and Effort, Prentice-Hall, Englewood Cliffs, NI, 1973.

[7] S.E. Kramer, C. Teunissen, A.A. Zekveld, Cortisol, chromogranin A, and pupillary responses evoked by speech recognition tasks in normally hearing and hard-of-hearing listeners: a pilot study, Ear Hear 37 (2016) 126S-135S.

[8] C.L. Mackersie, N. Calderon-Moultrie, Autonomic nervous system reactivity during speech recognition tasks: heart-rate variability and skin conductance, Ear Hear 37 (2016) 118S-125S.

[9] W. Klimesch, EEG alpha and theta oscillations reflect cognitive and memory performance: a review and analysis, Brain Res. Brain Res. Rev. 29 (1999) 169-195.

[10] J. Obleser, M. Wöstmann, N. Hellbernd, A. Wilsch, B. Maess, Adverse listening conditions and memory load drive a common $\alpha$ oscillatory network, J. Neurosci. 32 (2012) 12376-12383.

[11] G. Cartocci, A.G. Maglione, G. Vecchiato, G. Di Flumeri, A. Colosimo, A. Scorpecci, P. Marsella, S. Giannantonio, P. Malerba, G. Borghini, P. Arico, F. Babiloni, Mental workload estimations in unilateral deafened children, in: Engineering in Medicine and Biology Society (EMBC), IEEE, 2015, pp. 1654-1657, 37th Annual International Conference of the IEEE.

[12] E.B. Petersen, M. Wöstmann, J. Obleser, S. Stenfelt, T. Lunner, Hearing loss impacts neural alpha oscillations under adverse listening conditions, Front. Psychol. 6 (2015) 177.

[13] G. Cartocci, A.G. Maglione, D. Rossi, E. Modica, P. Malerba, F. Babiloni, The influence of different cochlear implant features use on the mental workload index during a word in noise recognition task, Int. J. Bioelectromagn. 18 (2016) 60-66.

[14] A.G. Maglione, G. Cartocci, D. Rossi, E. Modica, P. Malerba, G. Borghini, P. Aricò, G. Di Flumeri, F. Babiloni, Cochlear implant features and listening effort induction: measurement of the mental workload experienced during a word in noise recognition task, society for applied neuroscience conference 2016, held in Corfu, Front. Hum. Neurosci. (6-8 October 2016), http://dx.doi.org/ 10.3389/conf.fnhum.2016.220.00043.

[15] R.J.M. Somsen, B.J. Van-Klooster, M.W. Van-der-Molen, H.M. Van-Leeuwen, Growth spurts in brain maturation during middle childhood as indexed by EEG power spectra, Biol. Psychol. 44 (1997) 187-209.

[16] M. Turrini, F. Cutugno, P. Maturi, S. Prosser, F.A. Leoni, E. Arslan, Bisyllabic words for speech audiometry: a new Italian material, Acta Otorhinolaryngol. Ital. 13 (1993) 63-77.

[17] T.Y. Ching, R. Massie, E. Van Wanrooy, E. Rushbrooke, C. Psarros, Bimodal fitting or bilateral implantation? Cochlear Implants Int. 10 (2009). S23-S7.

[18] T.Y. Ching, J. Day, P. Van Buynder, S. Hou, V. Zhang, M. Seeto, L. Burns, C. Flynn, Language and speech perception of young children with bimodal fitting or bilateral cochlear implants, Cochlear Implants Int. 15 (2014) S43-S46.

[19] K. Kokkinakis, N. Pak, Binaural advantages in users of bimodal and bilateral cochlear implant devices, J. Acoust. Soc. Am. 135 (2014) 47-53.

[20] T.E. Nichols, A.P. Holmes, Nonparametric permutation tests for functional neuroimaging: a primer with examples, Hum. Brain Mapp. 15 (2002) 1-25.

[21] P. Anderer, R.D. Pascual-Marqui, H.V. Semlitsch, B. Saletu, Electrical sources of P300 event-related brain potentials revealed by low resolution electromagnetic tomography: effects of normal aging, Neuropsychobiology 37 (1998) $20-27$.

[22] R.D. Pascual-Marqui, D. Lehmann, T. Koenig, K. Kochi, M.C. Merlo, D. Hell, M. Koukkou, Low resolution brain electromagnetic tomography (LORETA) functional imaging in acute, neuroleptic-naive, first-episode, productive schizophrenia, Psychiatry Res. 90 (1999) 169-179.

[23] D. Pizzagalli, R.D. Pascual-Marqui, J.B. Nitschke, T.R. Oakes, C.L. Larson, $\underline{\text { H.C. Abercrombie, S.M. Schaefer, J.V. Koger, R.M. Benca, R.J. Davidson, Anterior }}$ cingulate activity as a predictor of degree of treatment response in major depression: evidence from brain electrical tomography analysis, Am. J. Psychiatry 158 (2001) 405-415.

[24] A.G. Srinivasan, M. Padilla, R.V. Shannon, D.M. Landsberger, Improving speech perception in noise with current focusing in cochlear implant users, Hear Res. 299 (2013) 29-36.

[25] N. Zhou, B.E. Pfingst, Effects of site-specific level adjustments on speech recognition with cochlear implants, Ear Hear 35 (2014) 30-40.

[26] P. Sauseng, W. Klimesch, What does phase information of oscillatory brain activity tell us about cognitive processes? Neurosci. Biobehav. Rev. 32 (2008) $1001-1013$.

[27] J. Onton, A. Delorme, S. Makeig, Frontal midline EEG dynamics during working memory, Neuroimage 27 (2005) 341-356.

[28] W. Klimesch, B. Schack, P. Sauseng, The functional significance of theta and upper alpha oscillations for working memory: a review, Exp. Psychol. 52 (2005) 99-108.

[29] M.Z. Zakrzewska, A. Brzezicka, Working memory capacity as a moderator of load-related frontal midline theta variability in Sternberg task, Front. Hum. Neurosci. 8 (2014) 399.

[30] M.G. Wisniewski, E.R. Thompson, N. Iyer, J.R. Estepp, M.N. Goder-Reiser, S.C. Sullivan, Frontal midline $\theta$ power as an index of listening effort, Neuroreport 26 (2015) 94-99.

[31] S.P. Kelly, E.C. Lalor, R.B. Reilly, J.J. Foxe, Increases in alpha oscillatory power reflect an active retinotopic mechanism for distracter suppression during sustained visuospatial attention, J. Neurophysiol. 95 (2006) 3844-3851.

[32] A.C. Snyder, J.J. Foxe, Anticipatory attentional suppression of visual features indexed by oscillaory alpha-band power increases: a high-density electrical mapping study, J. Neurosci. 30 (2010) 4024-4032.

[33] T.A. Rihs, C.M. Michel, G. Thut, Mechanisms of selective inhibition in visual spatial attention are indexed by a-band EEG synchronization, Eur. J. Neurosci. 25 (2007) 603-610.

[34] D. Cosmelli, V. López, J.P. Lachaux, J. López-Calderón, B. Renault, J. Martinerie, F. Aboitiz, Shifting visual attention away from fixation is specifically associated with alpha band activity over ipsilateral parietal regions, Psychophysiology 48 (2011) 312-322.

[35] J.J. Foxe, G.V. Simpson, S.P. Ahlfors, Parieto-occipital approximately $10 \mathrm{~Hz}$ activity reflects anticipatory state of visual attention mechanisms, Neuroreport 9 (1998) 3929-3933.

[36] J.J. Foxe, A.C. Snyder, The role of alpha-band brain oscillations as a sensory suppression mechanism during selective attention, Front. Psychol. 2 (2011) $1-13$.

[37] K.M.G. Fu, J.J. Foxe, M.M. Murray, B.A. Higgins, D.C. Javitt, C.E. Schroeder, Attention-dependent suppression of distractor visual input can be crossmodally cued as indexed by anticipatory parieto-occipital alpha-band oscillations, Cogn. Brain Res. 12 (2001) 145-152.

[38] S. Banerjee, A.C. Snyder, S. Molholm, J.j. Foxe, Oscillatory alpha-band mechanisms and the deployment of spatial attention to anticipated auditory and visual target locations: supramodal or sensory-specific control mechanisms? J. Neurosci. 31 (2011) 9923-9932.

[39] M.G. Wisniewski, Indices of effortful listening can be mined from existing electroencephalographic data, Ear Hear 38 (2016) e69-e73.

[40] O. Jensen, J. Gelfand, J. Kounios, J.E. Lisman, Oscillations in the alpha band (9-12 Hz) increase with memory load during retention in a short-term memory task, Cereb. Cortex 12 (2002) 877-882.

[41] J. Kaiser, T. Heidegger, M. Wibral, C.F. Altmann, W. Lutzenberger, Alpha synchronization during auditory spatial short-term memory, Neuroreport 18 (2007) 1129-1132

[42] S. Haegens, D. Osipova, R. Oostenveld, O. Jensen, Somatosensory working memory performance in humans depends on both engagement and disengagement of regions in a distributed network, Hum. Brain Mapp. 31 (2010) $26-35$.

[43] F. Roux, P.J. Uhlhaas, Working memory and neural oscillations: alpha-gamma versus theta-gamma codes for distinct WM information? Trends Cogn. Sci. 18 (2014) 16-25.

[44] A. Strauss, M. Wöstmann, J. Obleser, Cortical alpha oscillations as a tool for auditory selective inhibition, Front. Hum. Neurosci. 8 (2014) 1-7.

[45] W.J. Hudspeth, K.H. Pribram, Stages of brain and cognitive maturation, J. Educ. Psychol. 82 (1990) 881-884. 